\title{
USO DE ANTIPSICÓTICOS ATÍPICOS EM PACIENTES COM ESQUIZOFRENIA**
}

\author{
Aline Ferreira Campos Correia ${ }^{{ }^{*}}$, Ivânia Vera ${ }^{2}$, Roselma Lucchese ${ }^{2}$, Graciele
} Cristina Silva ${ }^{3^{*}}$, Cybelle Coimbra ${ }^{4}$

1 - Farmacêutica. Mestranda no Programa de Mestrado em Gestão Organizacional, Universidade Federal de Goiás (UFG), Regional Catalão. Catalão- GO, Brasil,

(aline.farma.bio@hotmail.com)

2 - Docente da Universidade Federal de Goiás (UFG), Regional Catalão. Catalão - GO, Brasil

3*- Nutricionista, Universidade Federal de Goiás (UFG), Regional Catalão. CatalãoGO, Brasil

4 - Graduanda em Enfermagem, Universidade Federal de Goiás (UFG), Regional Catalão, Catalão- GO, Brasil

**Projeto de Pesquisa "Análise da Atenção em Saúde Mental e constituição da rede no Sistema Único de Saúde no Sudeste Goiano"

Recebido em: 08/04/2017 - Aprovado em: 10/06/2017 - Publicado em: 20/06/2017 DOI: 10.18677/EnciBio_2017A137

Os índices de obesidade têm aumentado em todo mundo, sobretudo em pacientes esquizofrênicos que fazem uso de medicamentos antipsicóticos atípicos. Entretanto, seus efeitos adversos ainda são pouco conhecidos. Trata-se de uma revisão integrativa $(\mathrm{RI})$ que objetivou sistematizar o conhecimento científico produzido acerca do uso de antipsicóticos atípicos em pacientes com esquizofrenia. Os critérios de inclusão foram artigos científicos na íntegra e originais, publicados entre os anos de 2006 a 2016, nos idiomas inglês, espanhol ou português. Utilizou-se os Descritores em Ciências da Saúde (DeCS) "mental health", "antipsychotic agents", "overweight", nas bases de dados da CAPES, LILACS, MedLine e Pubmed, ocorrida no mês de junho e julho de 2016. Resultou na análise e extração das informações de 17 artigos. Houve prevalência de estudos epidemiológicos. Os artigos analisados descreveram a associação dos antipsicóticos atípicos em pacientes com esquizofrenia, com diversas comorbidades, dentre elas o aumento de peso, que desencadeia o agravamento da saúde dessa população. O tratamento medicamentoso por meio dos antipsicóticos atípicos, apresentam resultados substanciais na minimização dos sintomas da esquizofrenia, porém alguns efeitos decorrentes dessa classe farmacológica, precisam ser melhor estudados em promoção a saúde dos pacientes esquizofrênicos.

PALAVRAS-CHAVE: agentes antipsicóticos, aumento de peso, saúde mental; 


\title{
ATYPICAL ANTIPSYCHOTICS USE IN PATIENTS WITH SCHIZOPHRENIA: INTEGRATIVE REVIEW
}

\begin{abstract}
The obesity rates had increased in worldwide, especially in schizophrenic, patients in atypical antipsychotic medicine use. However, its adverse effects are poorly explained. This integrative review (RI) aimed to systematize the scientific 4 knowledge produced about the use of atypical antipsychotics in patients with schizophrenia. The inclusion criteria were scientific articles full and original, published between the years 2006-2016, in English, Spanish or Portuguese. We used the Health Sciences Descriptors (DeCS) "mental health," "antipsychotic agents", "overweight" in the databases of CAPES, LILACS, MedLine and PubMed, which occurred between June and July 2016. Resulted the analysis and extraction of information of 17 articles. The most prevalence were epidemiological studies. The analyzed articles described the association of atypical antipsychotics in patients with schizophrenia, with several comorbidities, among them, the increase in weight, which triggers the worsening health of this population. Medicine treatment by atypical antipsychotics, have substantial results in reducing symptoms of schizophrenia, but some effects of this pharmacologic class, need to be better studied in promoting the health of schizophrenic patients.
\end{abstract}

KEYWORDS: antipsychotic agents, mental health, overweight

\section{INTRODUÇÃO}

A obesidade no mundo duplicou desde a década de 1980. Mais de 1,9 bilhões de pessoas com idade acima dos 18 anos, em 2014, estavam acima do peso, destes, 600 milhões eram obesos. Definida como depósito excessivo de gordura corporal e distribuição visceral, a obesidade pode impactar negativamente na saúde. Sua presença possibilita o desenvolvimento de doenças crônicas, o que aumenta significativamente a morbimortalidade (WHO, 2016a).

$O$ estudo da obesidade vem se difundindo na contemporaneidade, diante de muitos aspectos, sobretudo da Saúde Pública. Estudos prévios indicam que os pacientes obesos têm maior probabilidade de desenvolver transtornos mentais (SILVA et al., 2015a). Dentre estes, a esquizofrenia afeta mais de 21 milhões de pessoas no mundo, sendo uma das doenças mentais mais comum, podendo afetar as relações pessoais e laborais do indivíduo. Pode surgir precocemente no sexo masculino, o que explica o fato de existirem 12 milhões de homens com o diagnóstico da doença comparado ao sexo oposto (9 milhões), (WHO, 2016b).

Os transtornos da esquizofrenia retratam uma associação de "sintomas positivos", ou seja, característicos da própria doença, conhecidos como surtos psicóticos, que podem ou não se apresentar com sintomas de catatonia, delírios, alucinações, comportamento psicomotor anormal, impossibilidade de permanecer ou se manter sentado (acatisia) além da insensatez, e desordem no discurso (DSMV, 2014; WHO, 2016b).

As crises podem ser agudas ou múltiplas, com ou sem remissão, no curso da doença e podem ser seguidas também pelos denominados "sintomas negativos", como dificuldade na demonstração dos sentimentos, indiferença, retraimento social e uma sensação intensa de desânimo, que exacerba disposições ao autoextermínio (GIRALDI \& CAMPOLIM, 2014). O tratamento da esquizofrenia indicado é o uso de psicofármacos de segunda geração, ou seja, os antipsicóticos atípicos cujo 
mecanismo de ação está na atuação da minimização dos sintomas positivos e sintomas negativos (RANG et al.,2012). No entanto, o uso farmacoterapêutico destas substâncias pode causar efeitos indesejáveis, como alterações no perfil antropométrico. O aumento de peso é resultado da interação entre o fármaco em questão e os receptores histaminérgicos $\mathrm{H} 1$, contudo, os mecanismos de ação ainda são pouco conhecidos (SCHMITZ et al., 2015). As discrasias sanguíneas, como hiperglicemia e dislipidemias também estão presentes em exames bioquímicos de pessoas que usam este tipo de farmacoterápico, sobretudo, o aumento no perfil lipídico em geral (MANGURIAN et al., 2015).

Cabe salientar que, dentre as comorbidades envolvendo 0 uso de antipsicóticos atípicos, como o excesso de peso, obesidade abdominal, síndrome metabólica, doenças cardiovasculares e até a morte precoce já foram descritas em estudos nesses indivíduos (MANGURIAN et al., 2015). Pessoas com esquizofrenia têm 2 a 2,5 vezes mais chances de morrerem precocemente, comparadas à população geral (WHO, 2016a). Neste sentido, esta revisão integrativa objetivou sistematizar o conhecimento científico produzido acerca do uso de antipsicóticos atípicos em pacientes com esquizofrenia.

\section{MATERIAL E MÉTODOS}

O método de estudo foi uma revisão integrativa (RI) que compreendeu a apreciação de estudos substanciais que dão estruturas para a deliberação e a ascensão da prática clínica. Permite a sinopse da situação de determinada temática, sobre as lacunas em um campo de conhecimento, a partir da produção científica. Os achados podem ser utilizados por diversos profissionais da saúde, de forma crítica, de modo a conduzirem a prática clínica na tomada de decisão (MENDES et al., 2008).

A metodologia contemplou seis passos descritos a seguir: 1)identificação do tema; 2)elaboração dos critérios de inclusão e exclusão dos estudos; 3)seleção das informações a serem extraídas, bem como a categorização dos estudos; 4) leitura dos textos selecionados de acordo com os critérios de inclusão; 5) interpretação dos resultados e, 6)apresentação da síntese do conhecimento produzido (MENDES et al., 2008). A questão norteadora deste estudo foi: "Quais as contribuições dos estudos em evidenciar a relação do uso de antipsicóticos atípicos, bem como suas alterações metabólicas e sobrepeso, na Esquizofrenia"?

Os critérios de inclusão definidos foram: artigos científicos na íntegra e originais, publicados entre os anos de 2006 a 2016, nos idiomas inglês, espanhol ou português. Os textos deveriam abordar a problemática do uso de antipsicóticos e seus efeitos adversos, no título, resumo e corpo do texto. Os artigos repetidos nas bases de dados foram excluídos.

As informações de interesse foram extraídas após leitura dos artigos, baseando-se em um instrumento validado no país. Extraiu-se informações acerca da autoria, título, ano, país de origem da publicação, base de dados em que se encontrava o artigo, delineamento do estudo, nível de evidência, instrumentos utilizados, resultados e síntese das conclusões (URSI, 2005).

Os níveis de evidência foram separados em nível I: resultado de metanálise de estudos clínicos controlados e com randomização; nível II: estudos de desenho experimental; nível III: pesquisas quase experimentais; nível IV: estudos não experimentais, descritivos ou com abordagem metodológica qualitativa; nível V: relatórios de casos ou relatos de experiências; nível VI: opiniões de especialistas 
baseadas em normas ou legislação (STETLER et al.,1998).

Utilizou-se os Descritores em Ciências da Saúde (DeCS) e os Medical Subject Headings (Mesh) da National Library, utilizados foram "mental health", "antipsychotic agents", 'overweight", nas bases de dados da Coordenação de Aperfeiçoamento de Pessoal de Nível Superior, (CAPES), Literatura LatinoAmericana e do Caribe em Ciências da Saúde (LILACS); Medical Literature Analysis and Retrieval Sistem on line (MedLine), National Institutes of Health's National Library of Medicine (NIH/NLM) Pubmed. A busca foi efetuada por três pesquisadores de forma independente nos meses de junho e julho de 2016, conforme exposto na Figura 1.

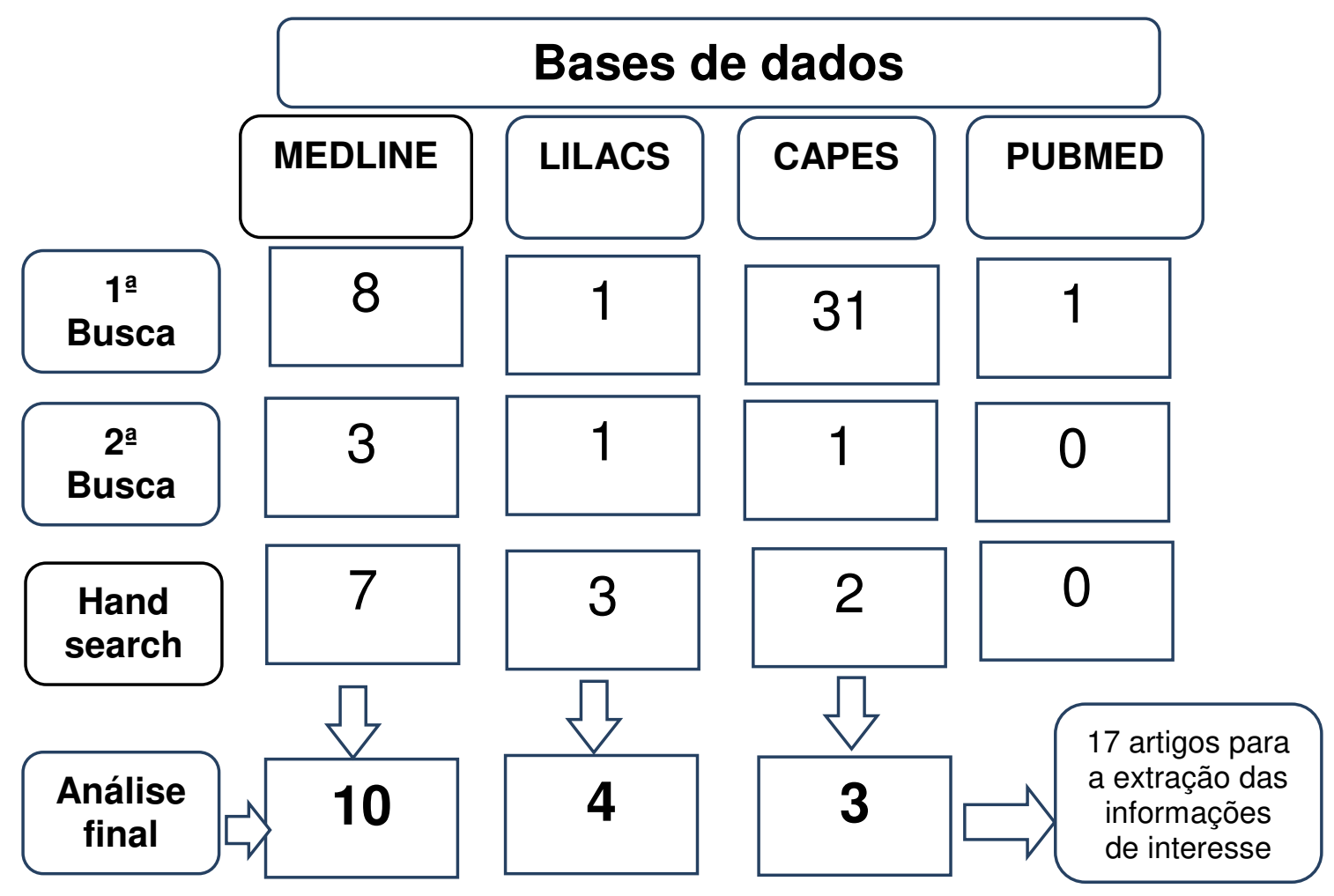

FIGURA 1: Diagrama de fluxo do processo de seleção dos artigos da amostra, 20062016. Fonte: As autoras, (2016). 
QUADRO 1: Perfil Antropométrico e Metabólico de pacientes com esquizofrenia em uso de antipsicóticos atípicos

\begin{tabular}{|c|c|c|c|}
\hline $\begin{array}{l}\text { Autores/ Ano/ Base de } \\
\text { dados/ País }\end{array}$ & Título & $\begin{array}{c}\text { Delineamento do estudo/ Nível de } \\
\text { evidência/ Instrumentos/ } \\
\text { Amostra }\end{array}$ & Principais Resultados \\
\hline $\begin{array}{l}\text { 1) DE HERT, et } \\
\text { al./2006/ Elsevier B.V } \\
\text { LILACS/ Belgium }\end{array}$ & $\begin{array}{l}\text { Body weight and self-esteem in } \\
\text { patients with schizophrenia evaluated } \\
\text { with B- WISE } \AA\end{array}$ & $\begin{array}{l}\text { Seccional/ Nível IV/ A Dutch translation } \\
\text { of B-WISE } \AA /(n=300)\end{array}$ & $\begin{array}{l}\text { B-WISE scores were positively associated } \\
\text { with BMI, metabolic syndrome and glucose } \\
\text { changes. Os patients with higher BMI had } \\
\text { less psychosocial adaptation. }\end{array}$ \\
\hline $\begin{array}{l}\text { 2) KWON, et al., } \\
\text { 2006/ MEDLINE/ } \\
\text { United States of } \\
\text { America (USA) }\end{array}$ & $\begin{array}{l}\text { Weight management program for } \\
\text { treatment-emergent weightgain in } \\
\text { olanzapine-treated patients with } \\
\text { schizophrenia or schizoaffective } \\
\text { disorder: A 12-week randomized } \\
\text { controlled clinical trial. }\end{array}$ & $\begin{array}{l}\text { Control case/ Nível III/ BMI, Abnormal } \\
\text { Involuntary Movement Scale (AIMS)/ } \\
\text { Vital signs (blood pressure, pulse rate), } \\
\text { laboratory data hematology, lipid } \\
\text { profile, and } \backslash \text { urinalysis/ }(\mathrm{n}=48)\end{array}$ & $\begin{array}{l}\text { The weight reduction in patients with } \\
\text { schizophrenia or schizoaffective disorder } \\
\text { taking olanzapine occurred at week } 8 \text { and } \\
\text { was also found to be safe in terms of } \\
\text { psychiatric symptoms, vital signs, and } \\
\text { laboratory data. }\end{array}$ \\
\hline $\begin{array}{l}\text { 3) CENTORRINO et } \\
\text { al./2006/ CAPES/ USA }\end{array}$ & $\begin{array}{l}\text { Weight loss in over weigh tpatients } \\
\text { maintained on atypic a lantipsychotic } \\
\text { agents }\end{array}$ & $\begin{array}{l}\text { Cross-sectional/ Nível IV/BMI, pulse, } \\
\text { blood pressure and Clinical Global } \\
\text { Impressions (CGI) in chronically } \\
\text { psychotic Patients/ }(n=17)\end{array}$ & $\begin{array}{l}\text { By } 24 \text { weeks, weight-loss/patient averaged } \\
6.0 \mathrm{~kg}(5.7 \%) \text { and BMl decreased to } 34.5 \text { (by } \\
5.7 \%) \text { Blood pressure decreased from } \\
130 / 83 \text { to } 116 / 74(11 \% \text { improvement), pulse }\end{array}$ \\
\hline $\begin{array}{l}\text { 4) FAULKNER, et al., } \\
\text { 2007/ Elsevier B.V } \\
\text { LILACS/ Canadian }\end{array}$ & $\begin{array}{l}\text { Body mass index, waist } \\
\text { circumference and quality of life in } \\
\text { individuals with schizophrenia }\end{array}$ & $\begin{array}{l}\text { Cross seccional /Nível IV/BMI, WC and } \\
\text { Quality of Life questionnaire (the MOS } \\
\text { SF-12) with two dimensions: physical } \\
\text { component summary (PCS) and mental } \\
\text { component summary (MCS) } /(\mathrm{n}=90)\end{array}$ & $\begin{array}{l}\text { WC was significant predictor of dimension } \\
\text { PCS - Quality of life in schizophrenic } \\
\text { patients. }\end{array}$ \\
\hline $\begin{array}{l}\text { 5) WU et al., } \\
\text { 2007/CAPES/Taiwan }\end{array}$ & $\begin{array}{l}\text { Outcomes of obese, clozapine-treated } \\
\text { in patients with schizophrenia placed } \\
\text { on a six-month diet and physical } \\
\text { activity program. }\end{array}$ & 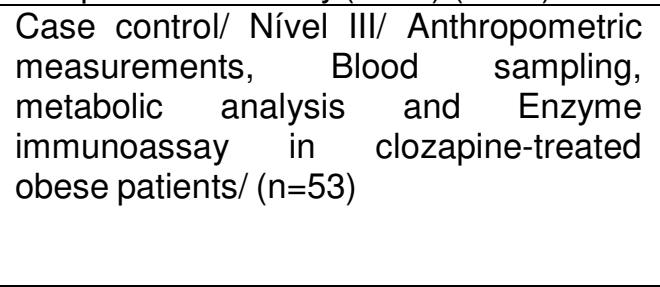 & $\begin{array}{l}\text { Compared with the control group, the study } \\
\text { group (A program diet with reduced calorie } \\
\text { intake by } 200 \text { to } 300 \text { kcal) decrease body } \\
\text { weight, BMI ( } 5.4 \% \text { reduction), WC (3.3 } \\
\mathrm{cm}) \text {, and hip circumference ( } 3.3 \mathrm{~cm} \text { ) after } \\
\text { three months and after six months. } \\
\text { Triglyceride and insulin-like growth factor- }\end{array}$ \\
\hline $\begin{array}{l}\text { 6)ZORTEA et al./ 2009/ } \\
\text { LILACS/ Brazil }\end{array}$ & $\begin{array}{lcr}\text { Avaliação } & \text { antropométrica } & \text { e } \\
\text { bioquímica em pacientes com } & \text { em pariona }\end{array}$ & $\begin{array}{l}\text { Cross-seccional/ Nível IV/ } \\
\text { BMI, CA, Percentual de gordura/( } n=40)\end{array}$ & $\begin{array}{l}\text { Pacientes tratados com clozapina } \\
\text { apresentam aumento de peso e significante } \\
\text { anormalidade lipídica, necessitando de } \\
\text { monitoramento frequente dos níveis } \\
\text { plasmáticos e intervenção nutricional }\end{array}$ \\
\hline
\end{tabular}




\begin{tabular}{|c|c|c|c|}
\hline $\begin{array}{l}\text { 7)KILBOURNE et al./ } \\
\text { 2009/ MEDLINE/ USA }\end{array}$ & $\begin{array}{l}\text { Excess heart-disease-related mortality in } \\
\text { a national study of patients with mental } \\
\text { disorders: Identifying modifiable risk } \\
\text { factors. }\end{array}$ & $\begin{array}{l}\text { Cross-seccional/ Nível IV/ } \\
\text { Date Large Health Survey of Veteran } \\
\text { Enrollees }(\text { LHSV }) /(n=147.193)\end{array}$ & $\begin{array}{l}\text { Patients with psychosis were more likely } \\
\text { to die from HD. For reduction of HD- } \\
\text { related mortality, early interventions that } \\
\text { promote smoking cessation and physical } \\
\text { activity among veterans with psychotic }\end{array}$ \\
\hline $\begin{array}{l}\text { 8)ZORTEA et al., 2010/ } \\
\text { LILACS/Brazil }\end{array}$ & $\begin{array}{l}\text { Estado nutricional de pacientes com } \\
\text { esquizofrenia frequentadores do Centro } \\
\text { de Atenção Psicossocial (CAPS) do } \\
\text { Hospital de Clínicas de Porto Alegre }\end{array}$ & $\begin{array}{l}\text { Cross-seccional/ Nível IV/ } \\
\text { Peso, BMI, WC e Percentual de } \\
\text { gordura/(n=40) }\end{array}$ & $\begin{array}{l}\text { Pacientes com esquizofrenia tiveram } \\
\text { aumentados WC, percentual de gordura } \\
\text { corporal e peso. }\end{array}$ \\
\hline $\begin{array}{l}\text { 9) DE WINTER, C.F. et al./ } \\
\text { 2010/ } \\
\text { Netherlands }\end{array}$ & $\begin{array}{l}\text { Metabolic syndrome in } 25 \% \text { of older } \\
\text { people with Intellectual disability (ID) }\end{array}$ & $\begin{array}{l}\text { Cross-sectional/ Nível IV/ WC, systolic } \\
\text { blood pressure, glucose, triglycerides } \\
\text { and }(H D L) /(n=470)\end{array}$ & $\begin{array}{l}\text { The prevalence of the metabolic } \\
\text { syndrome in the population with ID was } \\
\text { higher than in the general Dutch } \\
\text { population aged } \geq 50 \text { years }(15.7 \%, 95 \%\end{array}$ \\
\hline $\begin{array}{l}\text { 10)DRUSS, B.G. et al./2011/ } \\
\text { MEDLINE/ USA }\end{array}$ & $\begin{array}{l}\text { Understanding excess mortality in } \\
\text { persons with mental illness: } 17-\text { Year } \\
\text { follow up of a nationally representative } \\
\text { US survey. }\end{array}$ & $\begin{array}{l}\text { Cross-sectional/ Nível IV/ data from the } \\
1989 \text { National Health Interview Survey } \\
\text { mental health supplement, with } \\
\text { mortality data through/ } 2006 \text { linked } \\
\text { through the National Death Index /(n= } \\
80.850)\end{array}$ & $\begin{array}{l}\text { Persons with mental disorders died an } \\
\text { average of } \\
8.2 \text { years younger than the rest of the } \\
\text { population. }\end{array}$ \\
\hline 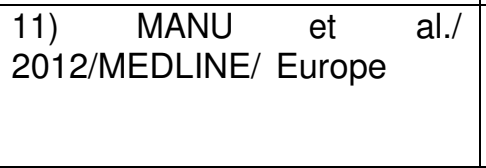 & $\begin{array}{l}\text { Prediabetes in patients treated with } \\
\text { antipsychotic drugs }\end{array}$ & $\begin{array}{l}\text { Cohort Studt/ Nível III/ BMI, WC, } \\
\text { glucose tolerance test, and fasting } \\
\text { insulin and lipids/(n=783) }\end{array}$ & $\begin{array}{l}\text { Prediabetes is highly prevalent in } \\
\text { adults treated with antipsychotic drugs } \\
\text { and correlates with markers of } \\
\text { increased intra abdominal adiposity, }\end{array}$ \\
\hline $\begin{array}{l}\text { 12) DAUMIT et al./ } \\
\text { 2013/ MEDLINE/ } \\
\text { Europe }\end{array}$ & $\begin{array}{l}\text { A behavioral weight-loss intervention in } \\
\text { persons with serious mental illness. }\end{array}$ & $\begin{array}{l}\text { Control group/ Nível III/ } \\
\text { Randomized Trial Of Achieving Healthy } \\
\text { Life styles in Psychiatric Rehabilitation } \\
\text { (ACHIEVE)/(n=291) }\end{array}$ & $\begin{array}{l}\text { A behavioral weight-loss intervention } \\
\text { significantly reduced weight over a } \\
\text { period of } 18 \text { months in overweight and } \\
\text { obese adults with serious mental illness. }\end{array}$ \\
\hline $\begin{array}{l}\text { 13) CRUMP et al./ 2013/ } \\
\text { MEDLINE/ Europe }\end{array}$ & $\begin{array}{l}\text { Comorbidities and mortality in persons } \\
\text { with schizophrenia: A Swedish national } \\
\text { cohort study. }\end{array}$ & $\begin{array}{l}\text { Cohort study/ Nível III/ the Swedish } \\
\text { Outpatient Registry and the Swedish } \\
\text { Hospital Registry/ }(n=6.097 .834)\end{array}$ & $\begin{array}{l}\text { On average, men with schizophrenia died } \\
15 \text { years earlier, and women } 12 \text { years } \\
\text { earlier, than the rest of the population, } \\
\text { and this was not accounted for by } \\
\text { unnatural deaths. The leading causes }\end{array}$ \\
\hline $\begin{array}{l}\text { 14) DE KUIJPER et al., } \\
2013 / \quad \text { MEDLINE/ } \\
\text { Netherlands }\end{array}$ & $\begin{array}{l}\text { Determinants of physical health } \\
\text { parameters in individuals with Intellectual }\end{array}$ & $\begin{array}{l}\text { Control group/ Nível III/ WC, weight, } \\
\text { height, systolic and diastolic blood } \\
\text { pressure, assessment of }\end{array}$ & $\begin{array}{l}\text { Extrapyramidal symptoms were present } \\
\text { in } 53 \% \text {, overweight or obesity in } 46 \% \text {, } \\
\text { and the metabolic Syndrome in }\end{array}$ \\
\hline
\end{tabular}

ENCICLOPÉDIA BIOSFERA, Centro Científico Conhecer - Goiânia, v.14 n.25; p.

16482017 


\begin{tabular}{|c|c|c|c|}
\hline & $\begin{array}{l}\text { disability who use long-term } \\
\text { antipsychotics. }\end{array}$ & $\begin{array}{l}\text { the presence and severity of } \\
\text { extra pyramidal symptoms/ } \\
(n=99) \text {. }\end{array}$ & $\begin{array}{l}\text { Hyperprolactineaemia and one or more } \\
\text { elevated bone turnover markers were present } \\
\text { in } 17 \% \text { and } 25 \% \text {, respectively. Higher age } \\
\text { and more severe intellectual } \\
\text { were associated with dyskinesia and a } \\
\text { higher dosage of the antipsychotic drug } \\
\text { was } \\
\end{array}$ \\
\hline $\begin{array}{l}\text { 15) MANU et al., 2013/ } \\
\text { MEDLINE/Belgium }\end{array}$ & 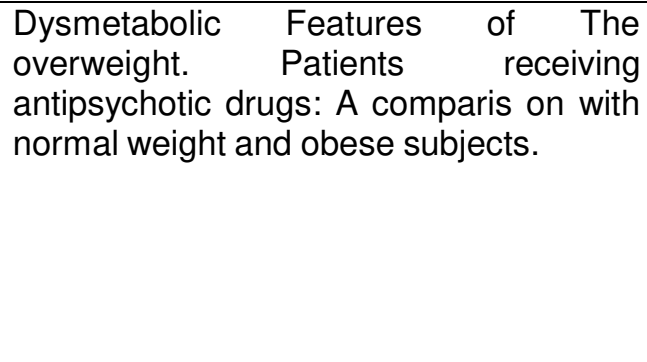 & $\begin{array}{l}\text { Cross-sectional/ Nível IV/ WC, oral } \\
\text { glucose tolerance test, and fasting } \\
\text { insulin and lipid }(n=820) \text {. }\end{array}$ & $\begin{array}{l}\text { Overweight patients }(n=212) \text { had higher fasting } \\
\text { insulin resistance as assessed with the } \\
\text { homeostatic model, insulin secretion during the } \\
\text { oral glucose tolerance test, triglycerides and } \\
\text { low- density lipoprotein cholesterol and lower } \\
\text { levels of high-density lipoprotein cholesterol. } \\
\text { The obese }(n=50) \text { were different from the } \\
\text { overweight subjects only with respect to } \\
\text { higher post-challenge insulin levels. }\end{array}$ \\
\hline $\begin{array}{l}\text { 16) GREEN et al./2014 / } \\
\text { MEDLINE/ USA }\end{array}$ & $\begin{array}{l}\text { A 12-Week Weight } \quad \text { Reduction } \\
\text { Intervention for Overweight Individuals } \\
\text { Taking Antipsychotic Medications. }\end{array}$ & $\begin{array}{l}\text { Case control/ Nível III/ Height, } \\
\text { weight, and blood pressure were } \\
\text { measured by blinded study staff/ } \\
(\mathrm{n}=36)\end{array}$ & $\begin{array}{l}\text { Mean weight in intervention participants } \\
\text { declined from } 213.3 \text { to } 206.6 \text { pounds, while } \\
\text { control participants' weight was unchanged. }\end{array}$ \\
\hline $\begin{array}{l}\text { 17) GREEN et al., 2015/ } \\
\text { CAPES/ USA }\end{array}$ & $\begin{array}{l}\text { The STRIDE Weight Loss and Life style } \\
\text { Intervention for Individuals taking } \\
\text { Antipsychotic } \\
\text { Randomized Trial. }\end{array}$ & $\begin{array}{l}\text { Control Case/ Nível III/ Height, } \\
\text { weight, BMI, Blood samples/ } \\
(n=190) \text {. }\end{array}$ & $\begin{array}{l}\text { Intervention participants lost } 4.4 \mathrm{~kg} \text { more than } \\
\text { control participants from baseline to } 6 \text { months } \\
(95 \% \mathrm{Cl}=-6.96 \mathrm{~kg} \text { to }-1.78 \mathrm{~kg} \text { ) and } 2.6 \mathrm{~kg} \\
\text { more than control participants from baseline } \\
\text { to } 12 \text { months }(95 \% \mathrm{Cl}=-5.14 \mathrm{~kg} \text { to }-0.07 \mathrm{~kg}) \text {. } \\
\text { At } 12 \text { months, fasting glucose levels in the } \\
\text { control group had increased from } 106.0 \\
\mathrm{mg} / \mathrm{dL} \text { to } 109.5 \mathrm{mg} / \mathrm{dL} \text { and decreased in the } \\
\text { intervention group from } 106.3 \mathrm{mg} / \mathrm{dL} \text { to } 100.4\end{array}$ \\
\hline
\end{tabular}

Fonte: As autoras, (2016). 


\section{RESULTADOS E DISCUSSÃO}

A amostra deste estudo de $\mathrm{RI}$ resultou em 17 artigos científicos para a análise final, os quais estão expostos no diagrama 1. Destes, 10 artigos (58,83\%) na base de dados MEDLINE, 4 (23,53\%) na LILACS, $3(17,64 \%)$ na CAPES. Em relação à origem dos periódicos, os países são, em ordem crescente: Suíça (1/5,88\%); Taiwan (1/5,88\%); Canadá (1/5,88\%); Europa (2/11,77\%); Bélgica (2/11,77\%); Brasil (2/11,77\%); Holanda (2/11,77\%); Estados Unidos da América (EUA) (6/35,28\%).

Quanto à abrangência da revista científica, predominaram as publicações internacionais com 15 artigos (88,23\%), e dois artigos nacionais (11,77\%). Esses achados podem ser justificados, pelo fato de a MEDLINE, sendo um componente do PubMed e uma das bases de dados de acesso livre com indexação de mais de cinco mil periódicos dos EUA e de mais de 80 países, apresentar interface nas áreas de ciências da saúde (MEDLINE, 2016).

A maioria das indústrias farmacêuticas está localizada geograficamente na América Central. Essas indústrias corroboram para o fato na identificação quanto à origem dos artigos serem em sua maioria internacionais e na língua inglesa. A estas estão relacionadas o maior número de patentes conferidas, bem como um investimento financeiro na pesquisa e no desenvolvimento de novos fármacos (AKKARI et al., 2016).

$\mathrm{Na}$ análise do nível de evidência (STETLER et al.,1998), verificou-se delineamento transversal na maioria dos textos selecionados (9/52,94\%); casocontrole $(6 / 35,29 \%)$, seguida pela metodologia coorte $(2 / 17,77 \%)$. Assim, o nível de evidência encontrada nos textos selecionados, comprova que houve metodologias pujantes, ao revelar que a maioria se origina de estudos epidemiológicos, dentre estes os estudos seccionais que buscam pela transversalidade e prevalências do fenômeno em estudo, contudo, não possibilitam a inferência de causa e efeito (KLEIN \& BLOCH, 2009). Há de se ressaltar também, a metodologia do estudo de coorte, que dentro dos estudos observacionais permite monitorar os indivíduos avaliados e a incidência do fenômeno, permitindo a relação causa-efeito nos sujeitos do estudo (COELI \& FAERSTEIN, 2009).

Em relação ao ano de publicação, 2013 foi o mais predominante nos achados dessa RI. Isso talvez se deve ao fato dos profissionais da área da psiquiatria que estudam sobre a esquizofrenia, terem padronizado o uso do Manual Diagnóstico e Estatístico de Transtornos Mentais (DSMV), uma vez que antes, em todos os pacientes usava-se o diagnóstico de Transtorno Mental (TM) (DSMV, 2014). Foi no início da primeira década do século XXI que houve o crescimento dos antipsicóticos $\mathrm{n}$ a indústria farmacêutica (MACHADO \& FERREIRA, 2014).

Todos os artigos selecionados nessa RI foram produção científica de pesquisadores da área médica, sobretudo da área da psiquiatria. Em relação aos anos de publicação, destacam-se: 2013 , com quatro artigos (23,52\%); o ano de 2006 com tres $(17,64 \%)$ e, os anos de 2007,2009 e 2010, todos com do is artigos (11,76\%), seguidos por 2015, 2014, 2012 e 2011, com um (5,8\%\%). Não houve publicações no ano de 2008 , de acordo com os critérios de inclusão instituídos para esta revisão integrativa. 
$\mathrm{Na}$ apreciação dos artigos selecionados, verificou-se a correlação das comorbidades acometidas pelo uso de antipsicóticos em grupos heterogêneos em relação ao sexo e faixa etária, na qual a maior prevalência foi em adultos de ambos os sexos. As comorbidades mais encontradas foram o aumento do peso corporal, dislipidemias, hiperglicemia, propensão à síndrome metabólica e riscos cardiovasculares.

Sobre as evidências do aumento de peso corporal e uso de antipsicóticos atípicos (KWON et al., 2006; ZORTEA et al., 2010; MANU et al., 2013), há evidências que as medicações antipsicóticas se mostram como um fator de risco considerável quanto ao aumento de peso, sobretudo em pessoas sedentárias ou que realizam pouca atividade física (VANCAMPFORT et al., 2016).

O controle do peso corporal por dieta (KWON et al., 2006), mudanças no estilo de vida (WU et al., 2007; ZORTEA et al., 2009; DAUMIT et al., 2013), bem como, melhoria na qualidade de vida dos usuários de antipsicóticos atípicos (FAULKINER et al., 2007; GREEN et al., 2014; GREEN et al., 2015) são fatores preponderantes encontrados nesta RI. Uma orientação é que pacientes com diagnóstico de esquizofrenia devem fazer atividades físicas com modalidade de resistência ou treinamento concorrente com vistas à melhora na força e hipertrofia muscular e saúde funcional, compreendidas em, no mínimo duas vezes por semana para obtenção de benefícios de ordem física e mental (SILVA et al., 2015b).

Mudanças relacionadas aos hábitos alimentares, associadas a exercícios físicos, redução do ato tabagístico também podem contribuir na promoção da saúde (PARLETTA et al., 2016). Quanto às mudanças comportamentais e estilo de vida, os fatores de risco para doenças cardíacas estão entre os achados nesse estudo. Destacam-se o infarto do miocárdio (KILBOURNE et al., 2009; ZORTEA et al., 2010; CRUMP et al., 2013) e hipertensão arterial (DE KUIJPER et al., 2013), como principais fatores de risco para usuários de antipsicóticos atípicos.

Entre os pacientes doentes mentais que fazem uso dessas medicações, os determinantes de alterações na vascularização cardíaca necessitam de precauções clínicas específicas. Contudo, estudos com metodologias mais robustas se fazem necessários para conhecimento das implicações da farmacoterapia de antidepressivos e antipsicóticos em relação aos motivos de intercorrências cardiovasculares (PÉREZ-PIÑAR et al., 2016).

Em relação à qualidade de vida $(\mathrm{QV})$ do indivíduo que faz uso de antipsicóticos atípicos, (FAULKINER et al., 2007; GREEN et al., 2014; GREEN et al., 2015), algumas alterações adversas podem comprometê-la. Dentre estas, citam-se os sintomas positivos, pensamento desorganizado e défict de suporte social. Acrescenta-se, a depressão que precisa ser monitorada (SUTTAJIT \& PILAKANTA, 2015). Programas de intervenções não farmacológicas como mudanças no estilo de vida e comportamental além da gestão de peso, dentro de limites recomendados (WHO, 2016b), são mudanças significativas que têm o potencial para restringir o ganho de peso associado com medicamentos usados para tratar distúrbios psiquiátricos e melhorar a QV desses pacientes (PROVENCHER et al., 2016).

Quanto ao uso de instrumentos descritos nas metodologias dos artigos 
selecionados, diversos instrumentos são citados. Desde índex que abordaram as consequências psicossociais subjetivas (B-WISE) (DE HERT et al., 2006) e questionário de qualidade de vida (CENTORRINO et al., 2006; FALKNER et al., 2007).

Para mensuração do perfil antropométrico, os artigos descrevem uso de índice de massa corpórea, peso e circunferência abdominal (CENTORRINO et al., 2006; KWON et al., 2006; ZORTEA et al., 2009; GREEN et al., 2014; GREEN et al.,

2015). A antropometria (peso corpóreo em quilogramas [kg] e circunferência da cintura) é uma medida padronizada na identificação e categorização do peso e classificação dos indivíduos (WHO, 2016a). O índice de massa corporal (IMC) é uma medida simples obtida pela divisão do peso $(\mathrm{kg})$ multiplicado pela altura ${ }^{2}$ (altura $x$ altura), comumente usado para classificar o sobrepeso $\left(\geq 25 \mathrm{~kg} / \mathrm{m}^{2}\right)$ e obesidade $\left(\geq 30 \mathrm{~kg} / \mathrm{m}^{2}\right)$, para ambos os sexos em indivíduos adultos (WHO, 2016a).

Os principais exames de sangue incluem análise do perfil lipídico, hematológico e análise da glicemia (KWON et al., 2006; WU et al., 2007; MANU et al., 2011; MANU et al., 2013). Conhecer o perfil hematológico do paciente, bem como os índices glicêmicos, faz parte do rastreamento para a síndrome metabólica, ocasionando maiores chances do indivíduo desenvolver cardiopatias, acidente vascular encefálico e diabetes (SBEM, 2016).

Em continuidade as comorbidades, a síndrome metabólica também foi relatada nessa pesquisa (DE WINTER et al., 2010; MANU et al., 2011; DE KUIJPER et al., 2013). A síndrome metabólica tem sido prevalente em indivíduos sedentários, com excesso de peso, além do fator genético, ou seja, histórico de diabetes familiar, hiperglicemia e hipertensão arterial (SBEM, 2016). Em relação ao diabetes mellitus, o uso das medicações antipsicóticas precisa ser considerado na escolha da farmacoterapia no tratamento da esquizofrenia, tomando-se por base os fatores de risco para a ocorrência (VANCAMPFORT et al., 2016).

A urinálise foi descrita em apenas uma metodologia (KWON et al., 2006). O teste tem como indicações: avaliar a função tubular renal, detectar precocemente a disfunção renal, bem como diagnosticar o diabetes, além de analisar a capacidade renal de concentração urinária. Em relação aos antipsicóticos atípicos, os mais citados foram a Olanzapina (KWON et al., 2006) e Clozapina (WU et al., 2007; ZORTEA et al., 2009), sendo os demais artigos selecionados nessa RI, com discussão ampla sobre a classe de antipsicóticos atípicos. A utilização da Olanzapina no tratamento farmacológico contínuo da esquizofrenia evidencia efeitos contra as manifestações de hostilidade, sendo esse, um dos motivos de sua escolha (VOLAVKA et al., 2016). O uso da Clozapina foi correlacionado a índices reduzidos de internações psiquiátricas, suspensão da farmacoterapia (STROUP et al.,2015).

\section{CONCLUSÃO}

Esta $\mathrm{RI}$ revelou que a maioria das publicações está em idioma inglês, disponíveis na íntegra na base de dados da América Latina. Os principais instrumentos utilizados para mensuração do perfil antropométrico foram o IMC e circunferência abdominal e os bioquímicos, prevaleceram os níveis 
glicêmicos e testes de resistência à insulina, cujas alterações acima dos limites tolerados inclinam para a síndrome metabólica, uma comorbidade associada ao aumento do peso corporal e uso de drogas antipsicóticas em pacientes diagnosticados com esquizofrenia. Os riscos cardiovasculares e piora na qualidade de vida também foram observados e merecem aprofundamento por meio de metodologias mais robustas.

\section{REFERÊNCIAS}

AKKARI, A.C.S.; TOMIOKA, J.; SANTOS, N.M.B.F.; SANTOS, R.F. Pharmaceutical innovation: differences between Europe, USA and 'pharmerging'countries. Gestão \& Produção, n. AHEAD, p. 0-0, 2016. Disponível em: <http://dx.doi.org/10.1590/0104-530X2150-15>. DOl:10.1590/0104- 530X2150-15.

CENTORRINO, F.; WURTMAN, J.J.; DUCA, K.A.; FELLMAN, V.H.; FOGARTY, K.V.; BERRY, J.M.; GUAY, D.M.; ROMELING, M.; KIDEWELL, J.M.; CINCOTTA, S.L.; BALDESSARINI, R.J. Weight loss in overweight patients maintained on atypical antipsychotic agents. International Journal of Obesity, v. 30, n. 6, p. 1011-1016, 2006. Disponível em: < http://dx.doi.org/10.1038/sj.ijo.0803222>. DOI:10.1038/sj.ijo.0803222.

COELI, C. C.; FAERSTEIN, C. M. Estudos de Coorte. In: MEDRONHO, R. A. Epidemiologia. 2. ed. Rio de Janeiro: Atheneu.2009. cap. 12.

CRUMP, C.; WINKLEBY, M.A.; SUNDQUIST, K.; SUNDQUIST, J. Comorbidities and mortality in persons with schizophrenia: a Swedish national cohort study.American Journal of Psychiatry, v. 170, n. 3, p. 324-333, 2013. Disponível em:<http://dx.doi.org/10.1176/appi.ajp.2012.12050599>.DOI:10.1176/appi.ajp.2012. 12050599.

DAUMIT, G.L.; DICKERSON, F.B.; WANG, N.; DALCIM, A.; JEROME, G.J.; ANDERSON, C.; et al.,; A behavioral weight-loss intervention in persons with serious mental illness. New England Journal of Medicine, v. 368, n. 17, p. 15941602, 2013. Disponível em: <hhtp://dx.doi.org/10.1056/NEJMoa1214530>. DOI: 10.1056/NEJMoa1214530.

DE HERT, M.; PEUSKENS, B.; WINKEL, R.V.; KALNICKA, D.; HANSSSENS,L.; EYCK, D.V.; et al. Body weight and self-esteem in patients with schizophrenia evaluated with B-WISE®. Schizophrenia research,

v. 88, n. 1, p. 222-226, 2006. Disponível em: < hhtp://dx.doi.org/ 10.1016/j.schres.2006.07.025>. DOI:10.1016/j.schres.2006.07.025.

DE KUIJPER, G.; MULDER, H.; EVENHUIS, H.; SCHOLTE, F.; NISSER, F.; HOEKSTRA, P.J. Determinants of physical health parameters in individuals with intellectual disability who use long-term antipsychotics. Research in developmental disabilities, v. 34, n. 9, p. 2799-2809, 2013. Disponível em: <hhtp://dx.doi.org/10.1016/j.ridd.2013.05.016>. DOI:10.1016/j.ridd.2013.05.016. 
DE WINTER, C.F.; MAGILSEN, K.W.; ALFEN, J.C.V.; WILLENSEN, S.P.; EVENHUIS, H. M. Metabolic syndrome in $25 \%$ of older people with intellectual disability. Family Practice, p. cmq079, 2010. Disponível em: <hhtp://dx.doi.org/10.1093/fampra/cmq079>. DOI:10.1093/fampra/cmq079.

DRUSS, B.G.; ZHAO, L.; ESENWEIN, S.V.; MORRATO, E.H.; MARCUS, S.C. Understanding excess mortality in persons with mental illness: 17-year follow up of a nationally representative US survey. Medical care, v. 49, n. 6, p. 599-604, 2011. Disponível em: <hhtp://dx.doi.org/10.1097/MLR.0b013e31820bf86e>DOI:10.1097/MLR.0b013e318 $20 \mathrm{bf} 86 \mathrm{e}$.

\section{DSMV - O Manual Diagnóstico e Estatístico de Transtornos Mentais} (Diagnostic and Statistical Manual of Mental Disorders - DSM) (Porto Alegre, RS, 2014). Disponível em: <http://c026204.cdn.sapo.io/1/c026204/cldfile/1426522730/6d77c9965e17b15/b37dfc58aad8cd477904b9bb2ba8a75b/obau doeducador/2015/DSM\%20V.pdf>. Acesso em: 16 de set.2016.

FAULKNER, G.; COHN, T.; REMINGTON, G.; IRVING, H. Body mass index, waist circumference and quality of life in individuals with schizophrenia. Schizophrenia research, v. 90 , n. 1, p. 174-178, 2007. Disponível em: <hhtp://dx.doi.org/10.1016/j.schres.2006.10.009 >.DOI:10.1016/j.schres.2006.10.0 09.

GIRALDI, A.; CAMPOLIM, S. Novas abordagens para esquizofrenia. Ciência e Cultura , São Paulo, v. 66, n. 2, June 2014. Available from http://cienciaecultura.bvs.br/scielo.php?script=sci_arttext\&pid=S0009$67252014000200003 \& \operatorname{lng}=e n \& n r m=i s o>$. access on 16 Sept. 2016.

GREEN, C.A.; JANOFF, S.L.; YARBOROUGH, B.J.; YARBOROUGH, M.T. A $12-$ Week weight reduction intervention for overweight individuals taking antipsychotic medications. Community mental health journal, v. 50, n. 8, p. 974-980, 2014. Disponível em: <hhtp://dx.doi.org/10.1007/s10597-014-9716-9>. DOI:10.1007/s10597-014-9716-9.

GREEN, C.A.; YARBOROUGH, B.J.H.; LEO, M.C.; YARBOROUGH, M.T.; STUMBO, S.P.; JANOFF, S.L.; et al.,; The STRIDE weight loss and lifestyle intervention for individuals taking antipsychotic medications: a randomized trial. American Journal of Psychiatry, v. 172, n. 1, p. 1-21, 2015. Disponível em: <hhtp://dx.doi.org/10.1176/appi.ajp.2014.14020173>.DOI:10.1176/appi.ajp.2014.14 020173.

KILBOURNE, A.M.; MORDEN, N.E.; AUSTIN, K.; ILGEN, M.; MACCARTHEY, J.F.; DALACK, G.; BLOW, F.C. Excess heart-disease-related mortality in a national study of patients with mental disorders: identifying modifiable risk factors. General hospital psychiatry, v. 31, n. 6, p. 555-563, 2009. Disponível em: <hhtp://dx.doi.org/10.1016/j.genhosppsych.2009.07.008>.DOI:10.1016/j.genhospps ych.2009.07.008. 
KLEIN, C. H.; BLOCH, K. V. Estudos Seccionais. In: MEDRONHO, R. A. Epidemiologia. 2. ed. Rio de Janeiro: Atheneu. 2009. cap. 10.

KWON, J.S.; CHOI, J.S.; BAHK, W.M.; KIM, C.Y.; KIM, C.H.; SHIN. Y.C.; PARK, B.J.; OH, C.G. Weight management program for treatment-emergent weight gain in olanzapine-treated patients with schizophrenia or schizoaffective disorder: a 12week randomized controlled clinical trial. Journal of Clinical Psychiatry, v. 67, n. 4, p. 547-553, 2006. Disponível em: <hhtp://dx.doi.org/10.4088/JCP.v67n0405 DOI:10.4088/JCP.v67n0405.

MACHADO, L.V.; FERREIRA, R. R. A indústria farmacêutica e psicanálise diante da. Psicologia em Estudo, v. 19, n. 1, p. 135-144, 2014. Disponível em: <http://dx.doi.org/10.1590/1413-7372219160013>. DOI: 10.1590/14137372219160013.

MANGURIAN, C.; NEWCOMER J. W.; VITTINGHOFF, E.; CREASMAN, J.M.; KNAPP, P.; FUENTES-AFFLICK, E.; et al; Diabetes screening among underserved adults with severe mental illness who take antipsychotic medications. JAMA internal medicine. 2015;175(12):1977-1979. doi:10.1001/jamainternmed.2015.6098. Disponível em: <http://dx.doi.org/ 10.1001/jamainternmed.2015.6098>. DOI: 10.1001/jamainternmed.2015.6098

MANU, P.; CORRELL, C.U.; WANPERS, M.; WINKEL, R.V.; YU, D.; SHIFFELDRIM, D.; DE HEART, M. Dysmetabolic features of the overweight patients receiving antipsychotic drugs: a comparison with normal weight and obese subjects. European Psychiatry, v. 29, n. 3, p. 179-182, 2013. Disponível em: <hhtp://dx.doi.org/10.1016/j.eurpsy.2012.12.001>. 10.1016/j.eurpsy.2012.12.001

MANU, P.; CORRELL, C.U.; WINKEL, R.V.; WAMPERS, M.; DE HEART, M. Prediabetes in patients treated with antipsychotic drugs. The Journal of clinical psychiatry, v. 73 , n. 4, p. 460-466, 2011. Disponível em: <http://dx.doi.org/10.4088/JCP.10m06822>. DOI: 10.4088/JCP.10m06822.

MEDLINE Fact Sheet - National Library of Medicine. Disponível em: <https://www.nlm.nih.gov/pubs/factsheets/medline.html>. Acesso em: 16 set. 2016.

MENDES, K.D.S.; SILVEIRA, R.C.C.P.; GALVAO, C.M. Revisão integrativa: método de pesquisa para a incorporação de evidências na saúde e na enfermagem. Texto contexto - enferm. Florianópolis, v. 17, n. 4, p. 758-764, Dec. $2008 . \quad$ Available from < http://www.scielo.br/scielo.php?script=sci_arttext\&pid=S01040707200800040001 $8 \& \operatorname{lng}=$ en\&nrm=iso $>$. access on 07 Feb. 2017. http: // dx.doi.org/10.1590/S0104-07072008000400018>. DOI: 10.1590/S010407072008000400018.

PARLETTA N, ALJEESH Y, BAUNE BT. Health Behaviors, Knowledge, Life 
Satisfaction, and Wellbeing in People with Mental Illness across Four Countries and Comparisons with Normative Sample. Frontiers in Psychiatry. 2016;7:145.

PÉREZ-PIÑAR, M.; MATHUR, R.; FOGUET, Q.; AYIS, S.; J.; AYERBE, L. Cardiovascular risk factors among patients with schizophrenia, bipolar, depressive, anxiety, and personality disorders. European Psychiatry, v. 35, p. 8- 15, 2016. Disponível em: <http://dx.doi.org/10.1016/j.eurpsy.2016.02.004>. DOI: 10.1016/j.eurpsy.2016.02.004.

PROVENCHER, M.D.; BELANGER, M.E.; SHRIQUI, C.; LACHANCEC, I.; BONNEVILLE, S. L'éducation thérapeutique du patient pour les clientèles psychiatriques présentant un surpoids: l'exemple du programme «Wellness» développé au Québec. L'Encéphale, v. 42, n. 3, p. 201-207, 2016. Disponível em: http://dx.doi.org/10.1016/j.encep.2016.01.006>. DOI: 10.1016/j.encep.2016.01.006.

RANG. H.P.; DALE, M.N.; RYTTER, J.M.; FLOWER, R. J.; HENDERSON, G. Farmacologia. 7. ed. Rio de Janeiro: Elsevier, 2012. P. 534.

SCHMITZ, A. P.; KREUTZ, O. C.; SUYENAGA, E. S. Antipsicóticos atípicos versus efeito obesogênico sob a óptica da química medicinal. Revista Eletrônica de Farmácia, v. 12, n. 3, p. 23-35, 2015. Disponível em: <http://dx.doi.org/ 10.5216/ref.v12i3.33714>. DOI: http://dx.doi.org/10.5216/ref.v12i3.33714.

SILVA, T.M.; AGUIAR, O.B.; FONSECA, M.J.M. Associação entre sobrepeso, obesidade e transtornos mentais comuns em nutricionistas. Jornal Brasileiro de Psiquiatria. 2015a; 64(1):24-31. Disponível em: <htpp://dx.doi.org/10.1590/00472085000000053>. DOI: 10.1590/0047-2085000000053

SILVA, B. A.; CASSILHAS, R.C.; ATTUX, C.; CORDEIRO, Q.; GADELHA, A.L.; TELLES, B.A.; et al., ; A 20-week program of resistance or concurrent exercise improves symptoms of schizophrenia: results of a blind, randomized controlled trial. Revista Brasileira de Psiquiatria, v. 37, n. 4, p. 271- 279, 2015b. Disponível em: < http://dx.doi.org/10.1590/1516-4446-2014-1595>.DOI:10.1590/1516-4446-2014-1595.

SBEM - Sociedade Brasileira de Endocrinologia E Metabologia. A Síndrome Metabólica. Disponível em <http://www.endocrino.org.br/a-sindrome-metabolica/> Acesso em: 19 set. 2016.

STETLER, C, B; MORSI, D; RUCKI, S; BROUGHTON, S; CORRIGAN, B; FITZGERALD, J.; et al.,; Utilization-focused integrative reviews in a nursing service. Appl Nurs Res [Internet]. 1998[cited 2011 dez 17];11(4):195-206. Disponível em:<http://dx.doi.org/10.1016/S0897-1897(98)80329-7>. DOI:10.1016/S08971897(98)80329-7.

STROUP, T.S.; GERHARD, T.; CRYSTAL, S.; HUANG, C.; OLFSON, M. Comparative effectiveness of clozapine and standard antipsychotic treatment in adults with schizophrenia. American Journal of Psychiatry, v. 172, n. 3, p. 163173, 2015. Disponível em: <http://dx.doi.org/10.1176/appi.ajp.2015.15030332>. 
DOI:10.1176/appi.ajp.2015.15030332.

SUTTAJIT, S.; PILAKANTA, S. Predictors of quality of life among individuals with schizophrenia. Neuropsychiatric Disease and Treatment, v.11, p. 1371-1379, 2015. Disponível em: <http://dx.doi.org/10.2147/NDT.S81024>. DOI:10.2147/NDT.S81024.

URSI, ES. Prevenção de lesões de pele no perioperatório: revisão integrativa da literatura. [Dissertação]. Ribeirão Preto (São Paulo): Universidade de São Paulo Escola de Enfermagem de Ribeirão Preto; 2005. 128p. Disponível em:<http://dx.doi.org/10.11606/D.22.2005.tde-18072005-095456>. DOI: 10.11606/D.22.2005.tde-18072005-095456.

VANCAMPFORT, D. PROBS, M.; DAENEN, A.; DAMME, T. V.; DE HERT, M.; ROSENBAUM S.; BRUYNINCKX, D. Impact of antipsychotic medication on physical activity and physical fitness in adolescents: An exploratory study. Psychiatry Research, v. 242, p. 192-197, 2016. Disponível em: <http://dx.doi.org/ 10.1016/j.psychres.2016.05.042>. DOI: 10.1016/j.psychres.2016.05.042.

VOLAVKA, J.; DORN, R.A.V.; CITROME, L.; KAHN, R.S.; FLEISCHHACKER, W.W.; CZOBOR, P. Hostility in schizophrenia: An integrated analysis of the combined Clinical Antipsychotic Trials of Intervention Effectiveness (CATIE) and the European First Episode Schizophrenia Trial (EUFEST) studies. European Psychiatry, v. 31, p. 13-19, 2016. Disponível em: <http://dx.doi.org/10.1016/j.eurpsy.2015.10.003>. DOl: 10.1016/j.eurpsy.2015.10.003.

WHO - WORLD HEALTH ORGANIZATION (WHO). Obesity and overweight. Geneva: 2016a.

WHO - WORLD HEALTH ORGANIZATION (WHO). Schizophrenia. Geneva: 2016b.

WU, M.K.; WANG, C.K.; BAI, Y.M.; HUANG, C.Y.; LEE, S.D. Outcomes of obese, clozapine-treated inpatients with schizophrenia placed on a six-month diet and physical activity program. Psychiatric services, 2007. Disponível em: <http://dx.doi.org/10.1176/appi.ps.58.4.544>. DOI: 10.1176/appi.ps.58.4.544.

ZORTÉA, K.; BOCK, P.M.; MORENO, D.B.; ABREU, PSB. Avaliação antropométrica e bioquímica em pacientes com esquizofrenia usuários de clozapina. Revista de nutrição. Campinas, v. 22, n. 5 (set./out. 2009), p. 697705, 2009. Disponível em: <http://dx.doi.org/10.11606/issn.1806-6976.v11i4p224233>. DOI: 10.11606/issn.1806-6976.v11i4p224-233.

ZORTEA, K.; GUIMARÃES, L.R.; GAMA, C.S.; ABREU, P.S.B. Estado nutricional de pacientes com esquizofrenia frequentadores do Centro de Atenção Psicossocial (CAPS) do Hospital de Clínicas de Porto Alegre. Jornal Brasileiro de Psiquiatria , v. 59, n. 2, p. 126-130, 2010. Disponível em; <http://dx.doi.org/10.1590/S0047- 20852010000200008>. DOI:10.1590/S0047- 
20852010000200008. 